\title{
The Relationship between Serum 25(OH)D, PTH and Calculated HOMA-IR in Overweight Elderly Patients
}

\section{Hongfeng Jiang* and Shaorong Peng}

Gerontology Department, Puai hospital, Tongji Medical College, Huazhong University of Science and Technology, Wuhan, Hubei Province,430033, China

*Corresponding author: Jiang H, Gerontology Department, Puai hospital, Tongji Medical College, Huazhong University of Science and Technology, Wuhan, Hubei Province,430033, China, Tel: +86 027 68831380; E-mail: jhf2@sina.com

Received date: August 16, 2015; Accepted date: January 27, 2016; Published date: February 03, 2016

Copyright: (c) 2016 Jiang $\mathrm{H}$, et al. This is an open-access article distributed under the terms of the Creative Commons Attribution License, which permits unrestricted use, distribution, and reproduction in any medium, provided the original author and source are credited.

\begin{abstract}
Background: Vitamin D deficiency or insufficiency was commonly found among adults and particularly overweight elderly patients. It's controversial on the physiological roles of vitamin $D$ and parathyroid hormone (PTH) in metabolic diseases. We explored the relationship between serum 25-hydroxyvitamin D [25(OH)D], PTH and homeostasis model of assessment for insulin resistance index (HOMA-IR) in overweight elderly patients.

Methods: 162 elderly overweight patients were included as the overweight group, and 80 normal-weight elderly in-patients were enrolled as the control group. The concentrations of $25(\mathrm{OH}) \mathrm{D}, \mathrm{PTH}$, blood calcium (CA), fasting blood glucose (FBG), and fasting blood insulin (FINS) were determined and HOMA-IR were calculated.

Results: The occurrence rate of vitamin D deficiency or insufficiency, primary hypertension, diabetes mellitus type 2 and osteoporosis in the overweight group was significantly higher than that in the control group $(p<0.05)$. The levels of serum PTH, systolic blood pressure (SBP), diastolic blood pressure (DBP), waist circumference and HOME-IR of the overweight group were significantly higher than that of control group, while serum $25(\mathrm{OH}) \mathrm{D}$ and blood calcium were significantly lower $(p<0.05)$. The difference on serum phosphate levels was not significant. Body Mass Index (BMI) was positively correlated with HOMA-IR, $r=0.291(F=22.167, p<0.001)$; Serum 25(OH)D was negatively correlated with HOMA-IR, $r=-0.272(F=19.224, p<0.001)$; Serum PTH was positively correlated with HOMA-IR, $r=0.205(F=10.4883, p=0.001)$. Stepwise multiple regression analysis was applied and there was a correlation between HOMA-IR and BMI, 25(OH) D $(r=0.353, F=16.984, p<0.001)$.
\end{abstract}

Conclusion: Vitamin D deficiency or insufficiency and the increase of BMI were significantly associated with insulin resistance in overweight elderly patients.

Keywords: 25-hydroxyvitamin D; Parathyroid hormone; Insulin resistance; Overweight; Elderly people

\section{Introduction}

The obesity and overweight is becoming a common disease in the elderly in China. Hypertension, diabetes, hyperlipidemia and coronary artery disease are closely linked to obesity and overweight. It is more common that overweight is in the elderly in China. Many studies strongly suggested that the elderly adults of different genetic backgrounds existed lack of vitamin D status [1].Vitamin D deficiency or insufficiency was commonly found among adults and particularly among overweight patients.

The physiological roles of vitamin D and Parathyroid Hormone (PTH) in metabolic diseases are controversial. It has been reported that except for skeletal disease, rickets and osteomalacia, 25hydroxyvitamin $\mathrm{D}[25(\mathrm{OH}) \mathrm{D}]$ deficiency was also associated with cardiovascular diseases, obesity, metabolic syndrome and diabetes mellitus[2]. The research [3] by De Pergola G et al showed the level of $25(\mathrm{OH}) \mathrm{D}$ was significant independently associated with assessment for insulin resistance index (HOMA-IR). In contrast, no significant relationship between $\mathrm{PTH}$, vitamin $\mathrm{D}$ and metabolic syndrome has been shown in obese subjects [4]. Moreover, there was literature suggesting that $\mathrm{PTH}$, but not vitamin $\mathrm{D}$, was associated with the metabolic syndrome in the obese patients [5].

The objective of the present research was to explore the relationship between serum 25-hydroxyvitamin D, PTH and homeostasis model of assessment for insulin resistance index HOMA-IR in overweight elderly patients. The following research was carried out.

\section{Materials and Methods}

The study was performed at gerontology department of Puai hospital in Wuhan from January 2013 to June 2014. A total of 162 overweight elderly in-patients were enrolled in this study. All patients who participated in the study signed the informed consent prior to their participation in the research. The study protocol was approved by the Ethics Council of Puai hospital in Wuhan.

\section{Subjects}

The research subject included 84 males and 78 females with an average age of $76.6 \pm 8.8$ years, and the control subjects included 80 normal-weight elderly in-patients matched age and sex with the research subject, which included 42 males and 38 females with an average age of $75.9 \pm 7.8$ years. Those patients with an age under 60 years or patients with an abnormality of the kidney or liver were 
Page 2 of 5

excluded. Additionally, patients who had taken cholecalciferol supplementation were excluded.

The diagnostic criteria of obesity were based on the body mass index (BMI) standard recommended by The Guidelines for Prevention and Control of Overweight and Obesity in Chinese Adults, 2003. Normal weight subjects were required to have a BMI from $18.5 \mathrm{~kg} / \mathrm{m}^{2}$ to $23.9 \mathrm{~kg} / \mathrm{m}^{2}$, while overweight subjects were from $24 \mathrm{~kg} / \mathrm{m}^{2}$ to 27.9 $\mathrm{kg} / \mathrm{m}^{2}$ and obese subjects were over $28 \mathrm{~kg} / \mathrm{m}^{2}$. Those patients with a BMI from $24 \mathrm{~kg} / \mathrm{m}^{2}$ to $27.9 \mathrm{~kg} / \mathrm{m}^{2}$ were enrolled as subjects in the study.

\section{Detection of biochemical indicators}

Venous blood samples for the detection of serum 25hydroxyvitamin $\mathrm{D}[25(\mathrm{OH}) \mathrm{D}]$, parathyroid hormone $(\mathrm{PTH})$, fasting blood glucose (FBG) and fasting blood insulin (FINS) were drawn after a 12-hour fast and an empty stomach decubitus $30 \mathrm{~min}$ in the next morning. Blood samples were then collected for routine analysis and examination of liver and kidney function, blood lipids and other tests were conducted. Levels of serum $25(\mathrm{OH}) \mathrm{D}$ and serum PTH were measured by Wuhan center for Clinical laboratory (Wuhan, China) using electrochemiluminescent immunoassay (ECLIA) kit from Roche Diagnostics (Shanghai) Ltd. The intra-assay coefficient of variation for 25(OH)D and PTH were 5.2\% and 6.7\% respectively.

\section{Detection of anthropometric parameters and calculation of relative index}

The height and weight of participants were measured without shoes or hats and the subjects were accurate to $0.1 \mathrm{~kg}$ and $0.1 \mathrm{~cm}$; 2) Body Mass Index (BMI) $=$ Weight $/$ Height $^{2}$ (unit: $\mathrm{kg} / \mathrm{m}^{2}$ ) 0.3) The homeostasis model of assessment for insulin resistance index (HOMA-IR)=Fasting Blood Glucose $(\mathrm{FBG}) \times$ Fasting Blood Insulin $($ FINS)/22.5

\section{Statistical analysis}

All the data were analyzed by SPSS 11.5 software. The measurement data were presented as mean \pm standard deviation and mean comparison were done by independent-samples $\mathrm{T}$ test. Chi-square test was involved in the statistical analysis of the differences of count data and Pearson's correlation coefficient analysis was used to examine the relationship between two parameters. Two-sided $\mathrm{P}$ values $<0.05$ were considered statistically significant, and were not adjusted to account for multiple comparisons.

\section{Results}

\section{Comparison on clinical diseased of two groups}

Vitamin D deficiency was defined as serum 25(OH)D below 20 $\mathrm{ng} / \mathrm{mL}$, vitamin D insufficiency was between $20 \mathrm{ng} / \mathrm{mL}$ and $30 \mathrm{ng} / \mathrm{mL}$, and vitamin D sufficiency was more than $30 \mathrm{ng} / \mathrm{mL}$ [6]. Vitamin D deficiency and vitamin $\mathrm{D}$ insufficiency were the common diseases among Chinese elderly people recently. Our data suggested that the occurrence rate of Vitamin D deficiency or insufficiency in the overweight group was significantly higher than in the control group $(p<0.01)$, the same with the occurrence rate of primary hypertension, diabetes mellitus type 2 and osteoporosis in the overweight group $(p<0.05)$. This showed that the elderly overweight people have more metabolic syndrome relative disease (see Table 1).

\section{Comparison on BMI, blood pressure (BP), blood calcium (CA), blood phosphate (PHOS), serum 25(OH)D, PTH and HOMA-IR of two groups}

The level of serum PTH, waist circumference, both systolic blood pressure(SBP) and diastolic blood pressure (DBP) and HOME-IR of overweight elderly patients were significantly higher than that of controls, while the level of serum $25(\mathrm{OH}) \mathrm{D}$ and CA of overweight elderly patients were significantly lower than that of controls $(\mathrm{p}<0.05)$. Differences in blood phosphorus between the two groups did not reach statistical significance $(\mathrm{p}>0.05)$ (see Table 2$)$.

\begin{tabular}{|c|c|c|c|}
\hline Index & $\begin{array}{l}\text { Overweight group } \\
(n=162)\end{array}$ & $\begin{array}{l}\text { Control } \\
(n=80)\end{array} \quad$ group & ${ }^{*}$ P-value \\
\hline Male/Female & $84 / 78$ & $42 / 38$ & 0.924 \\
\hline $\begin{array}{l}\text { Vit } D \text { deficiency or Vit } \\
D \text { insufficiency }\end{array}$ & 131 & 47 & $<0.0001$ \\
\hline Primary hypertension & 102 & 37 & 0.0134 \\
\hline $\begin{array}{l}\text { Diabetes mellitus type } \\
2\end{array}$ & 92 & 33 & 0.0223 \\
\hline Osteoporosis & 74 & 20 & 0.0019 \\
\hline
\end{tabular}

Table1: Comparison on clinical disease of two groups.

\begin{tabular}{|c|c|c|c|c|c|c|c|c|c|}
\hline Group & BMI $\left(\mathbf{k g} / \mathbf{m}^{2}\right)$ & Waist (cm) & $\mathrm{BP}(\mathrm{mmHg})$ & $\begin{array}{l}25 \quad(\mathrm{OH}) \mathrm{D} \\
(\mathrm{ng} / \mathrm{ml})\end{array}$ & PTH (pg/mL) & HOMA-IR & PHOS (mmol/L) & $\mathrm{CA}(\mathrm{mmol} / \mathrm{L})$ & \\
\hline & & & SBP & DBP & & & & & \\
\hline Overweight group & $26.4 \pm 1.3$ & $99.6 \pm 7.0$ & $141.1 \pm 10.4$ & $77.6 \pm 8.2$ & $7.6 \pm 3.9$ & $52.6 \pm 22.3$ & $3.95 \pm 1.41$ & $1.08 \pm 0.18$ & $2.16 \pm 0.12$ \\
\hline Control group & $22.1 \pm 1.8$ & $81.0 \pm 6.8$ & $136.5 \pm 11.6$ & $73.4 \pm 8.1$ & $10.5 \pm 6.1$ & $45.8 \pm 16.8$ & $3.14 \pm 1.29$ & $1.11 \pm 0.19$ & $2.20 \pm 0.11$ \\
\hline T-value & 20.56 & 19.62 & 3.114 & 1.973 & 4.587 & 2.018 & 4.37 & 1.197 & 2.506 \\
\hline$p$-value & $<0.001$ & $<0.001$ & 0.0021 & 0.0498 & 0.001 & 0.045 & $<0.001$ & 0.2323 & 0.0129 \\
\hline \multicolumn{10}{|c|}{ Data were for 162 patients of overweight group and 80 patients of control group. } \\
\hline
\end{tabular}


Citation: Jiang H, Peng S (2016) The Relationship between Serum 25(OH)D, PTH and Calculated HOMA-IR in Overweight Elderly Patients.

$\mathrm{BMI}=$ body mass index; $\mathrm{BP}=$ blood pressure; $\mathrm{SBP}=$ systolic blood pressure; $\mathrm{DBP}=$ diastolic blood pressure; $\mathrm{PTH}=$ parathyroid hormone; HOMA-IR=homeostasis model of assessment for insulin resistance index; PHOS=Blood Phosphate; $\mathrm{CA}=$ Blood Calcium.

Table 2: Comparison of relative clinical index.

\section{Examination on the relationship between HOMA-IR and other variables by Pearson regression analysis}

Body Mass Index (BMI) and serum PTH concentration were positively correlated with HOMA-IR, $\mathrm{r}=0.291(\mathrm{~F}=22.167, \mathrm{p}<0.001)$ and $\mathrm{r}=0.205(\mathrm{~F}=10.4883, \mathrm{p}<0.001)$, and serum $25(\mathrm{OH}) \mathrm{D}$ was negatively correlated with HOMA-IR, $\mathrm{r}=-0.272(\mathrm{~F}=19.224, \mathrm{p}<0.001)$ (Figure 1-3).

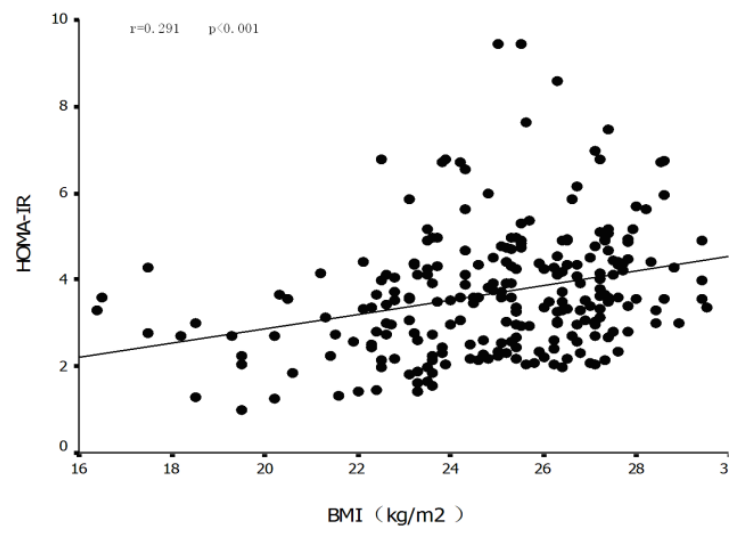

Figure 1: Correlations between BMI and HOMA-IR. BMI were positively correlated with HOMA-IR, $\mathrm{r}=0.291(\mathrm{~F}=22.167, \mathrm{p}<0.001)$.

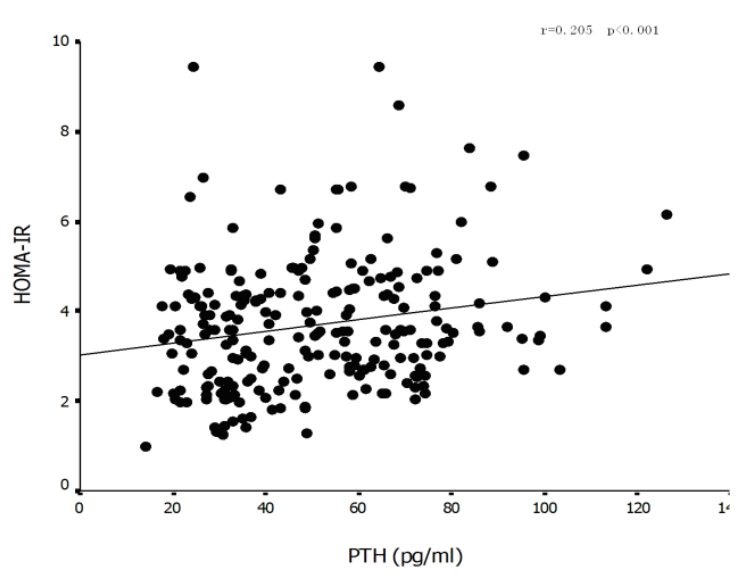

Figure 2: Correlations between HOMA-IR and serum PTH. PTH were positively correlated with HOMA-IR, $r=0.205$ ( $F=10.4883$, $\mathrm{p}<0.001)$.
Examination on the relationship between HOMA-IR and other variables by stepwise multiple regression analysis

We Selected the overweight elderly patients as the research subjects, and HOMA-IR was taken as variables, when age, sex, BMI, serum 25(OH)D, serum PTH, blood CA, blood PHOS and waist circumference were taken as parameter. $25(\mathrm{OH}) \mathrm{D}$ was associated with HOMA-IR and BMI in model 2, while there was no correlation between PTH and HOMAIR. There was a correlation between BMI, 25(OH)D and HOMA-IR in model $1(\mathrm{r}=0.353 \mathrm{~F}=16.984, \mathrm{p}<0.001)$. We found that vitamin $\mathrm{D}$ deficiency or insufficiency and the increase of BMI were significantly associated with insulin resistance in overweight elderly patients (see Table 3 ).

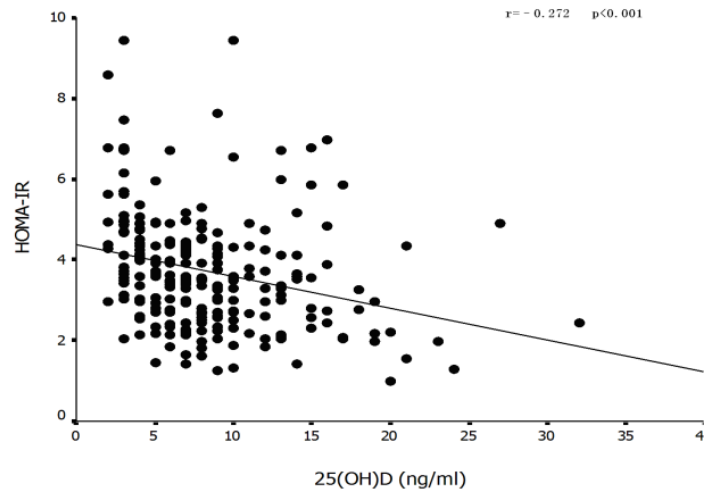

Figure 3: Correlations between serum 25(OH)D and HOMA-IR. Serum $25(\mathrm{OH}) \mathrm{D}$ was negatively correlated with HOMA-IR, $\mathrm{r}=-$ $0.272(\mathrm{~F}=19.224, \mathrm{p}<0.001)$.

\begin{tabular}{|l|l|l|l|l|l|l|}
\hline Index & B & $\begin{array}{l}\text { Std } \\
\text { error }\end{array}$ & Beta & t-value & p-value & $\mathbf{9 5 \% ~ C l ~ o f ~ B ~}$ \\
\hline Model1 C & 0.866 & 0.959 & & 0.903 & 0.368 & $(-1.024,2.756)$ \\
\hline 25(OH)D & -0.06 & 0.018 & -0.208 & -3.3 & 0.001 & $(-0.096,-0.024$ \\
\hline BMI & 0.134 & 0.036 & 0.233 & 3.705 & 0 & $(0.063,0.205)$ \\
\hline Model2 C & 0.801 & 0.962 & & 0.832 & 0.406 & $(-1.095,2.696)$ \\
\hline $25(\mathrm{OH}) \mathrm{D}$ & -0.059 & 0.018 & -0.205 & -3.249 & 0.001 & $(-0.095,-0.023$ \\
\hline BMI & 0.129 & 0.036 & 0.225 & 3.53 & 0 & $(0.057,0.201)$ \\
\hline PTH & 0.004 & 0.004 & 0.056 & 0.908 & 0.365 & $(-0.004,0.011)$ \\
\hline
\end{tabular}

Table3: Stepwise multiple regression analysis of HOMA-IR.

Data in Table 3 was obtained from overweight elderly patients. 


\section{Discussion}

Obesity and overweight in elderly people have become one of the most important factors of diabetes, hypertension, coronary disease and osteoporosis. According to the statistical data in 2003, nearly $25 \%$ of Chinese population was overweight or obese, and the disease rate of overweight elderly people in urban was $37.2 \%$, with a slightly higher rate among males than females [7].

This study found that older patients are widespread $25(\mathrm{OH}) \mathrm{D}$ deficiency and insufficiency, particularly in overweight elderly patients. Elderly patients are often suffering from various diseases, especially those overweight elderly patients whose willingness and ability to move positively was reduced, in addition with skin aging and decreasing of vitamin D synthesis. Therefore, vitamin D deficiency or insufficiency was due to ageing and lifestyle changes induced by ill health. Inflammatory processes involved in disease occurrence and clinical course would reduce $25(\mathrm{OH}) \mathrm{D}$, which would also explain why low vitamin $\mathrm{D}$ status was reported in a wide range of disorders.

We found that overweight elderly patients had a lower level of blood calcium and higher PTH than controls. Since the calcium synthesis and calcium absorption in the gastrointestinal tract and kidney tubules of elderly people are reducing, the level of blood calcium decreased and serum PTH increased correspondingly. That's why overweight or obese patients always have hyperparathyroidism. Meanwhile, vitamin $\mathrm{D}$ is important for good bone health since it's helpful on the absorption and utilization of calcium. Vitamin D deficiency or insufficiency in patients came with secondary hyperparathyroidism, increased phosphorus absorption and decreased blood calcium concertration. It resulted in increased bone resorption and high bone fragility and fracture risk. Recent studies indicated that hyperparathyroidism was linked to the increase of systolic blood pressure and diastolic blood pressure [8], and the correlations between serum PTH and blood pressure was approved in another research on Chinese elderly people [9]. The serum PTH of overweight elderly people was higher than those of normal-weight elderly people; therefore the occurrence rate of hypertension was higher in overweight elderly people.

In the present paper, we found that serum PTH was positively correlated with HOMA-IR. Low serum 25(OH)D concentrations are commonly found in the elderly and associated with compensatory hyperparathyroidism. Serum PTH was positively correlated with HOMA-IR, but no statistically significant interaction was observed between HOMA-IR and PTH by stepwise multi-regression analysis in overweight elderly patients. The reason might be that PTH had negative correlation with $25(\mathrm{OH}) \mathrm{D}$. Another reason was affected by extreme values. We cannot exclude the possibility that that overweight elderly patients might have introduced a selection bias.

Our study showed that BMI was positively correlative with HOMAIR. Because of the reduced blood calcium concentration in overweight elderly patients and the weakened calcium regulation, the energy metabolism enzyme did not convert the energy substance absorbed by the body into a supporting structure or ATP, and more carbohydrates and proteins converted into glucose in the blood, and then insulin resistance increased. Meanwhile, Lipid in the form of fat deposited on the surface of the skin and internal organs and developed metabolic syndrome. BMI and waist circumference increased, which is characterized by insulin resistance and diabetes increased. With successive elimination of multiple regression analysis, BMI was significantly associated with insulin resistance in elderly patient.
Our study showed a significantly higher HOMA-IR in overweight elderly patients than in controls, and serum 25(OH)D of elderly patient was negatively correlated with HOMA-IR. With stepwise multiple regression analysis, serum $25(\mathrm{OH}) \mathrm{D}$ was significantly associated with HOMA-IR. Peroxisome proliferator activated receptor (PPAR) $-\delta$ was able to adjust the metabolism of fatty acids in skeletal muscle and adipose tissue, thereby regulating insulin resistance. PPAR$\delta$ could be directly activated by vitamin $\mathrm{D}$ to improve insulin resistance status [10]. In the recent animal study [11], when serum $25(\mathrm{OH}) \mathrm{D}$ concentration in obese Wister rats model of diabetes mellitus type 2 increased, there was a decrease in the blood glucose and a rise of blood insulin which had no association with blood calcium. Meta-analysis of literature [12] suggested that pre-diabetes status could be improved by vitamin D supplement, including improvement on secretion of insulin, fasting insulin sensitivity index and peripheral insulin resistance. Some studies suggested vitamin $D$ was associated with insulin secretion of $G$ cell in pancreas since vitamin $\mathrm{D}$ receptors and vitamin $\mathrm{D}$ conjugated proteins were found on the surface of $G$ cell in pancreas. Meanwhile, animal study suggested that insulin secretion of rat would decrease for vitamin $\mathrm{D}$ deficiency and the increase of insulin secretion rely on the vitamin D supplement [13]. Therefore we considered Vitamin D deficiency or insufficiency as one risk factor of diabetes in the elderly overweight patients. This conclusion was consistent withthe existing literature $[3,14,15]$.

Many conflicting views were found on the relationship between vitamin $\mathrm{D}$ and type 2 diabetes in recent literatures. The relative evidence [16] also showed no effect of vitamin D supplementation on glucose homeostasis or diabetes prevention. Even though these studies with type 2 diabetes as research end point got the negative from ours, we still insisted that there was relationship between low vitamin D levels and type 2 diabetic, since diabetes prevalence factors were multiple and vitamin D deficiency or insufficiency was one of the factors. Since the type 2 diabetes mellitus was a slowly progressive disease, it was impractical to reduce the occurrence and development of diabetic by vitamin $\mathrm{D}$ supplementation in a relatively short period of time (less than 10 years). The lower 25(OH)D level might only be a marker of ill health [17]. Further research was required on this aspect. The study [18] from Husemoen LL found low serum 25(OH)D was inverse associated independently with incident diabetes and the association was only found in overweight-obese but not in normal weight individuals. hose literatures which denied the correlation between low vitamin $\mathrm{D}$ and diabetic were more or less mixed with normal weight individuals. Moreover, meta-analysis form Song Y[15] showed an inverse and significant association between circulating $25(\mathrm{OH}) \mathrm{D}$ levels and risk of type 2 diabetes.

Our research has limitations. First, the overweight elderly patients might have a selection bias. Second, there was no comparison in the relation between vitamin $\mathrm{D}$, parathyroid hormone and HOMA-IR in young patients. Finally, a logistic binary seems to be more appropriate.

\section{Conclusion}

According to our study, we found that low serum 25(OH)D levels and high serum PTH levels are in overweight elderly patients. We also found that HOMA-IR remained negatively related with serum $25(\mathrm{OH}) \mathrm{D}$ and positively related with BMI and serum PTH. The result of stepwise multiple regression analysis suggested that there was a correlation between BMI, 25(OH)D and HOMA-IR. Thus, we concluded that vitamin D deficiency or insufficiency as well as 
increased BMI are significantly associated with insulin resistance in overweight elderly patients.

\section{Acknowledgements}

Thanks for the help of Wuhan center for Clinical laboratory.Thank Caihua Huang and Jie Zhang for collection and registration of data. Caihua Huang and Jie Zhang are the physician in gerontology Department of Puai hospital.

\section{Author Contributions}

Conceived and designed the experiments: $\mathrm{H}$ Jiang, $\mathrm{S}$ Peng. Performed the experiments: H Jiang, S Peng. Contributed regents/ materials/analysis tools: $\mathrm{H}$ Jiang. Wrote the paper: $\mathrm{H}$ Jiang, S Peng.

\section{References}

1. Dawson-Hughes B, Mithal A, Bonjour JP, Boonen S, Burckhardt P, et al (2010) IOF position statement: vitamin D recommendations for older adults. Osteoporos Int 21: 1151-1154.

2. Holick MF (2004) Sunlight and vitamin D for bone health and prevention of autoimmune diseases, cancers, and cardiovascular disease. Am J Clin Nutr 80: 1678S-88S.

3. De Pergola G, Nitti A, Bartolomeo N, Gesuita A, Giagulli VA, et al. (2013) Possible role of hyperinsulinemia and insulin resistance in lower vitamin D levels in overweight and obese patients. Biomed Res Int 2013: 921348.

4. Rueda S, Fernández-Fernández C, Romero F, Martínez de Osaba J, Vidal J (2008) Vitamin D, PTH, and the metabolic syndrome in severely obese subjects. Obes Surg 18: 151-154.

5. Hjelmesaeth J, Hofsø D, Aasheim ET, Jenssen T, Moan J, et al. (2009) Parathyroid hormone, but not vitamin $\mathrm{D}$, is associated with the metabolic syndrome in morbidly obese women and men: a cross-sectional study Cardiovasc Diabetol 8: 7.

6. Mao X, Zheng H, Liu Z, Wu Y, Na R, et al. (2014) Analysis of 25(OH)D serum concentrations of hospitalized elderly patients in the Shanghai area. PLoS One 9: e90729.
7. Wu YF, Ma GS, Hu YH, Li YP, Li X, et al. (2005) [The current prevalence status of body overweight and obesity in China: data from the China National Nutrition and Health Survey]. Zhonghua Yu Fang Yi Xue Za Zhi 39: 316-320.

8. Mateus-Hamdan L, Beauchet O, Bouvard B, Legrand E, Fantino B, et al. (2013) High parathyroid hormone, but not low vitamin D concentrations, expose elderly inpatients to hypertension. Geriatr Gerontol Int 13: 783-791.

9. Chan R, Chan D, Woo J, Ohlsson C, Mellström D, et al. (2012) Serum 25hydroxyvitamin $\mathrm{D}$ and parathyroid hormone levels in relation to blood pressure in a cross-sectional study in older Chinese men. J Hum Hypertens 26: 20-27.

10. Ozfirat Z, Chowdhury TA (2010) Vitamin D deficiency and type 2 diabetes. Postgrad Med J 86: 18-25.

11. Santos RD, Vianna LM (2005) Effect of cholecalciferol supplementation on blood glucose in an experimental model of type 2 diabetes mellitus in spontaneously hypertensive rats and Wistar rats. Clin Chim Acta 358: 146-150.

12. Barengolts E (2010) Vitamin D role and use in prediabetes. Endocr Pract 16: 476-485.

13. Danescu LG, Levy S, Levy J (2009) Vitamin D and diabetes mellitus. Endocrine 35: 11-17.

14. Barchetta I, De Bernardinis M, Capoccia D, Baroni MG, Fontana M, et al. (2013) Hypovitaminosis D is independently associated with metabolic syndrome in obese patients. PLoS One 8: e68689.

15. Song Y, Wang L, Pittas AG, Del Gobbo LC, Zhang C, et al. (2013) Blood 25-hydroxy vitamin $\mathrm{D}$ levels and incident type 2 diabetes: a meta-analysis of prospective studies. Diabetes Care 36: 1422-1428.

16. Seida JC, Mitri J, Colmers IN, Majumdar SR, Davidson MB, et al. (2014) Effect of vitamin $\mathrm{d} 3$ supplementation on improving glucose homeostasis and preventing diabetes: a systematic review and meta-analysis. J Clin Endocrinol Metab 99: 3551-3560.

17. Autier P, Boniol M, Pizot C, Mullie P (2014) Vitamin D status and ill health: a systematic review. Lancet Diabetes Endocrinol 2: 76-89.

18. Husemoen LL, Skaaby T, Thuesen BH, Jørgensen T, Fenger RV, et al, (2012) Serum 25(OH)D and incident type 2 diabetes: a cohort study. Eur J Clin Nutr 66: 1309-1314. 